\title{
SEDIMENTOS URBANOS: AMBIENTE E ÁGUA
}

\section{URBAN SEDIMENTS: ENVIRONMENT AND WATER}

\section{Cristiano Poleto, Leidy Luz García Martinez}

\author{
${ }^{1}$ Universidade Tecnológica Federal do Paraná, Campus Toledo. Rua Cristo Rei, 19, \\ CEP: 85902-490 - Toledo, PR - Brasil \\ Telefone: (45) 33796800, e-mail: cristiano_poleto@hotmail.com \\ ${ }^{2}$ Instituto de Pesquisas Hidráulicas. Av. Bento Gonçalves, 9500 -Agronomia, CEP: \\ 91501-970 - Porto Alegre, RS - Brasil \\ Telefone: (51) 33086670. e-mail: luxgm@yahoo.es
}

\begin{abstract}
RESUMO
Os sedimentos urbanos são considerados, atualmente, como a maior fonte de poluição difusa para os corpos de água em áreas urbanas devido ao transporte de metais pesados e outros poluentes. Metais pesados são considerados potencialmente perigosos devido ao seu potencial tóxico e bioacumulativo, ainda que em pequenas concentrações. A dinâmica do processo de transporte de metais pelos sedimentos é bastante complexa e está sujeita a variáveis, tais como a distribuição granulométrica de partículas de sedimentos, a origem e suas fontes potenciais, além do tipo e ocupação da bacia hidrográfica. Por isso, é necessário entender os processos envolvidos na origemtransporte-deposição dos sedimentos em ambientes urbanos para poder caracterizar (quantidade e qualidade) essas cargas de poluentes que aportam nos corpos de água. Estes tipos de informações podem ser utilizados como uma forma de suporte para a concepção de projetos sustentáveis de drenagem urbana, que permitam minimizar a degradação da qualidade dos ambientes aquáticos.
\end{abstract}

Palavras-chave: Bacia hidrográfica urbana. Metais pesados. Granulometria. Poluição difusa.

\begin{abstract}
The dynamics of the metal's transport pathways by sediments is sufficiently complex and it is influenced for some variables such as the sediment size-grain distribution, origin and its potential sources as well the type and occupation of the urban watershed. Therefore, it's necessary to understand the involved processes in the sources-transport-deposit of the sediments in urban environments to be able to characterize (quantity and quality) these loads of pollutants that arrive in freshwater bodies. This kind of information can be used like support for sustainable projects conception of urban draining and it allows to minimize the degradation of the aquatic's environments quality.
\end{abstract}

Keywords: Urban watershed. Heavy metals. Size-grain distribution. Diffuse pollution. 


\section{INTRODUÇÃO}

Com o desenvolvimento das civilizações e o consequente crescimento populacional, cresceu também a utilização dos recursos hídricos disponíveis. Já há algum tempo, associa-se a urbanização à poluição dos corpos de água, devido aos esgotos domésticos não tratados e aos despejos industriais. Mais recentemente, no entanto, percebeu-se que nas áreas urbanas, parte desta poluição tem origem no escoamento superficial que traz consigo uma grande carga de sedimentos gerados pelo processo de urbanização, influenciada também, pelo uso e o tipo do solo, além das atividades antrópicas realizadas na bacia hidrográfica (POLETO e CHARLESWORTH, 2010).

$\mathrm{O}$ escoamento superficial da água, nesses locais, transporta o material disponível para os corpos de água, carreando, portanto, uma quantidade de sedimentos bastante significativa. Além disso, a impermeabilização do solo leva ao aumento das velocidades de escoamento, aumentando a capacidade de arraste, o que pode trazer como consequência o aumento das concentrações de sedimentos e a potencialização dos processos erosivos, gerando assim, maiores cargas poluidoras.

As redes de drenagem urbana são responsáveis pela veiculação dessas cargas e sabe-se, hoje, que se constituem em importantes fontes de degradação de rios, lagos e estuários (PORTO et al., 2004; DOTTO, 2006).

Entre os elementos traço mais comuns encontrados em ambientes urbanos e, que, atualmente estão sendo associados às partículas de sedimentos, encontra-se uma ampla variedade de metais, como o zinco, chumbo, cobre, níquel, cádmio e cromo, que são utilizados com frequência em atividades industriais e descartados sem controle algum na rede coletora de águas pluviais ou de esgotos. Além disso, esses metais podem alcançar espaços públicos através da remoção da poeira pelo vento e outros vetores. Outros contaminantes como hidrocarbonetos, bactérias e pesticidas também se encontram em bacias urbanas pelas diferentes ações inerentes às atividades sociais e econômicas desenvolvidas. No entanto, os metais pesados merecem atenção especial pelas propriedades bioacumulativas e biomagnificantes em organismos vivos, podendo originar grandes distúrbios em ecossistemas (POLETO e CASTILHO, 2008).

Este tipo de poluição, normalmente conhecida como poluição difusa (devido ao mecanismo de distribuição), está associado ao transporte desses metais adsorvidos nas partículas de sedimentos, ao longo da bacia hidrográfica até corpos de água receptores finais. Isso ocorre devido à falta de estruturas que captem e armazenem os sedimentos oriundos de áreas impermeáveis, da construção civil, das obras civis já concluídas, da vegetação existente, das ruas não-pavimentadas e áreas de solos desprotegidas e/ou descobertos (POLETO et al., 2009).

O estudo e a compreensão da dinâmica do processo de urbanização e a produção e qualidade dos sedimentos são de grande importância, pois servem de ponto de partida para a elaboração de medidas que visem à maximização do uso dos recursos hídricos disponíveis e à convivência harmônica com a população (PORTO et al., 2004; DOTTO, 2006; POGGIO et al., 2009).

O estudo de sedimentos urbanos através da perspectiva da sedimentologia ambiental é relativamente novo. As pesquisas nesta área originaram-se pela crescente preocupação sobre os efeitos das partículas urbanas em relação à saúde pública e que inicialmente foram focadas nas partículas depositadas em ruas e estradas (TAYLOR, 2007).

Este trabalho pretende fazer uma revisão das informações mais relevantes dentro da dinâmica dos processos associados às fontes e ao transporte de sedimentos em ambientes urbanos e a influencia destes no transporte de metais pesados. O entendimento desta temática vai ajudar a esclarecer os efeitos da qualidade das bacias hidrográficas urbanas e sua relação com os ambientes aquáticos. 


\section{GENERALIDADES SOBRE OS SEDIMENTOS URBANOS}

A composição de sedimentos que se acumulam entre ambientes sedimentários é um reflexo dos seguintes processos: fontes de sedimentos, transporte e deposição de sedimentos e os processos químicos que atuam no sedimento durante o processo de fonte-transporte-deposição entre a origem e o ambiente aquático. Os sedimentos podem vir de fontes naturais e/ou antropogênicas e, frequentemente, podem ser classificados em sedimentos minerais, orgânicos (biogênicos) e partículas de sedimentos de origem antropogênicas (TAYLOR, 2007).

Minerais de quartzo, feldspatos junto com outros minerais formam o componente primário de muitos sedimentos terrestres e marinhos. Os sedimentos orgânicos são formados por restos de esqueletos fósseis, os quais predominam em ambientes marinhos. No entanto, variações latitudinais alteram o tipo e as taxas de produção destes sedimentos. Além disso, em áreas urbanas, ocorre um grande aporte de matéria orgânica proveniente do lançamento de esgoto clandestino. Por último, partículas de origem antropogênica presentes em grandes quantidades em ambientes urbanos (formadas por partículas de vidro, partículas metálicas, resíduos de processos industriais e da construção civil), apresentam propriedades químicas e mineralógicas diferentes das partículas de sedimentos de fontes naturais, portanto, interagem de forma diferente dentro do ambiente (MARTINEZ, 2010).

Um componente importante dos sedimentos de origem antropogênica, ausentes durante o período pré-industrial, são os poluentes, os quais incluem metais pesados, elementos inorgânicos, nutrientes e compostos orgânicos, os quais podem ser de origem natural ou antropogênicas, com alta predominância desta última. Em geral, segundo Charlesworth e Lees (1999), um sedimento normalmente está conformado por:

- Água intersticial que preenche os espaços entre as partículas, equivale a cerca de volume $50 \%$ do sedimento;

- Material inorgânico, rochas, fragmentos de conchas e grãos resultantes da erosão natural do material da crosta terrestre;

- Material orgânico que ocupa pequeno volume, mas é um componente importante devido à característica de sorção e biodisponibilidade de muitos contaminantes;

- Materiais e/ou resíduos sólidos de origem antrópica.

Na Figura 1 (a), apresenta uma partícula de sedimento simplificada (frações de argila e areia) de origem natural, conformada basicamente por uma associação entre matéria orgânica e óxidos de ferro e manganês. Na Figura 1 (b), é apresentada uma fotografia, obtida através de técnicas de microscopia eletrônica, da fração de argila fina de partículas de sedimentos coletadas em uma bacia ao sul do Brasil. Nela podem observar-se argilominerais como a ilita e a esmectita.

$\mathrm{Na}$ formação de sedimentos urbanos ocorre uma grande e complexa mistura que pode se originar de fontes mais comumente encontradas em ambientes urbanos, ou mesmo importadas de outras áreas próximas ou ainda muito afastadas, podendo, portanto, ser derivados de diversas fontes naturais e antropogênicas (ROBERTSON et al., 2003; POLETO, 2007). Além disso, os sedimentos podem ser originados de restos de materiais da construção civil, esse tipo de sedimento pode causar desequilíbrios ecológicos, como por exemplo, a geração de uma super população de moluscos devido ao aumento das concentrações de cálcio disponível no ambiente (POLETO, 2007) ou também alterar a dinâmica trófica em ecossistemas lacustres pelo acúmulo de metais pesados em organismos (RULE et al., 2006). 
a)

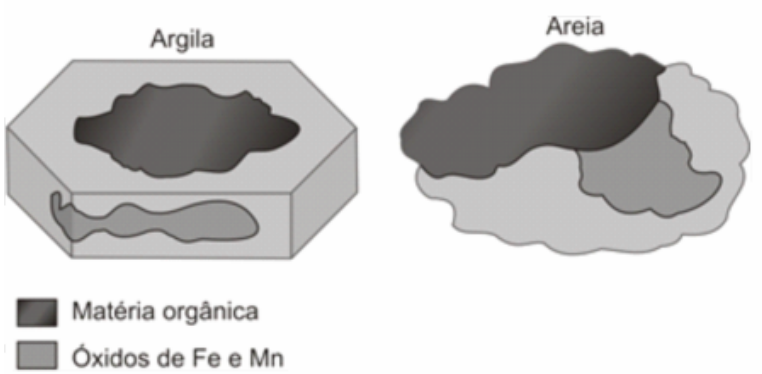

b)
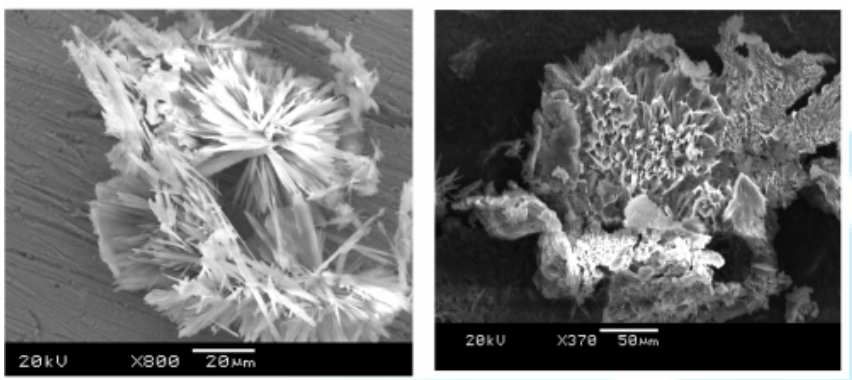

Figura 1. a) Estrutura simplificada de um grão de sedimento; b) Imagens de dois argilominerais obtidas por microscopia eletrônica de uma fração de argila fina de sedimentos coletados em uma bacia ao Sul do Brasil; (acima: ilita; embaixo: esmectita). Fotografias obtidas usando o equipamento Jeol ${ }^{\circledR}$ SEM.

Fontes: Federal Interagency Stream (1998); Bortoluzzi et al. (2006)

O reconhecimento da importância dos sedimentos como um constituinte fundamental na análise da qualidade da água vem crescendo nos últimos anos. A Agência de Proteção Ambiental dos Estados Unidos (EPA) identificou os sedimentos como o poluente mais comum nos rios e arroios americanos (GELLIS et al., 2003).

Os sedimentos são os poluentes potenciais mais importantes conduzidos pelo escoamento pluvial, o seu acúmulo provoca danos pela obstrução das canalizações, prejudicando o desempenho da rede de drenagem projetada. Além disso, os sedimentos carreados até o corpo receptor formam depósitos que vão assoreando o seu leito e, consequentemente, originando muitos problemas. Em resumo, as principais consequências da produção de sedimentos em áreas urbanas são:

- Obstrução da rede de drenagem;

- Redução da capacidade de escoamento de condutos, rios e lagos urbanos;

- Transporte de poluentes agregados ao sedimento que podem degradar os corpos de água.

O conhecimento da quantidade de sedimentos propagada ao longo da bacia até o exutório é de fundamental importância para o planejamento e o aproveitamento dos recursos hídricos de uma região, uma vez que, os danos causados pelos sedimentos dependem da quantidade e da natureza destes, os quais, por sua vez, dependem dos processos de erosão, transporte e deposição desses sedimentos. 


\section{FONTES DE SEDIMENTOS URBANOS}

As partículas de sedimentos urbanos podem ser originadas de uma ampla variedade de fontes, naturais e antrópicas (TAYLOR, 2007). Conforme os estudos realizados por Charlesworth e Lees (1999); Charlesworth et al. (2003); Gellis et al. (2003); Sutherland (2003); Al-Khashman (2004); Duzgoren-Aydin et al. (2006); Pereira et al. (2007); Poleto e Castilho (2008), as principais fontes de sedimentos urbanos são: materiais decorrentes da superfície de estradas e de sua manutenção, veículos automotores, lavagem de áreas adjacentes (permeáveis e impermeáveis), áreas de construção civil, atividades comerciais e industriais, resíduos sólidos, resíduos da vegetação, esgoto, solos junto às estruturas de microdrenagem, telhados e deposição atmosférica (Figura 2).

Para Gellis et al. (2003), as fontes de sedimentos urbanos mudam durante o crescimento do processo de urbanização. As fontes iniciais de sedimentos estão associadas a atividades da construção que ocasionam distúrbios na superfície do solo. Depois que estes locais em desenvolvimento atingem certa estabilização, a carga de sedimento liberada aos cursos de água é reduzida.

As taxas de produção de sedimentos do período de pós-desenvolvimento comumente são menores que aquelas presentes na condição de pré-desenvolvimento. Nelson e Booth (2002) concluíram que, para o caso da bacia Issaquah Creek (Washington, USA), a taxa de sedimentos aumentou em torno de $200 \mathrm{Kg} \cdot \mathrm{Ha}^{-1}$.ano ${ }^{-1}$ com o aumento da urbanização.

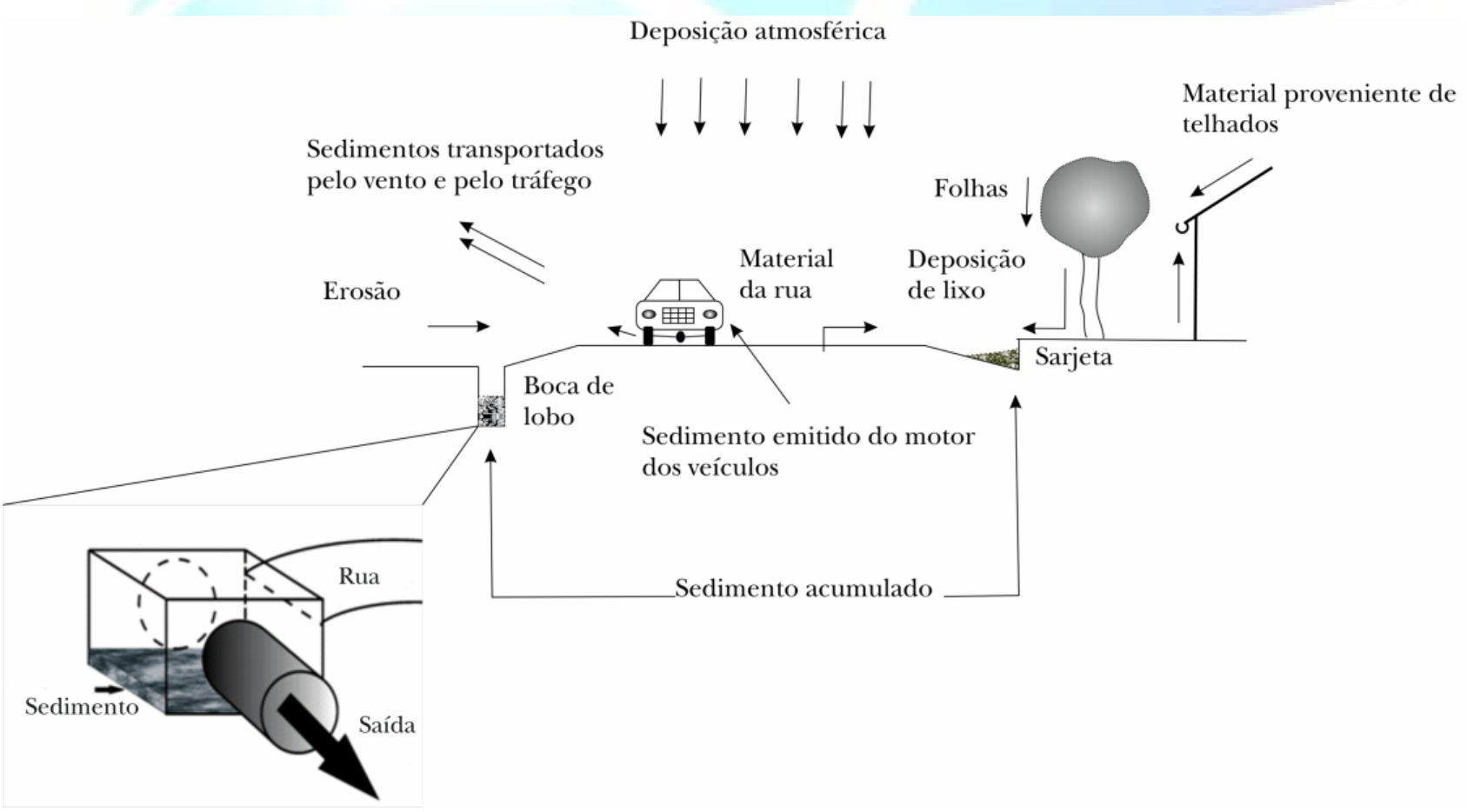

Figura 2. Fontes de sedimentos em ambientes urbanos.

Adaptado de: Dotto (2006); Poleto et al. (2009)

Nelson e Booth (2002) estudaram o balanço de sedimentos na supracitada bacia, avaliando as diferentes fontes e quantidades de sedimentos produzidos por diversos processos erosivos na montante e que estão continuamente sendo agravados por atividades antrópicas. Os valores de 
produção de sedimentos em distintas áreas urbanas são apresentados na Tabela 1. Destaca-se que a produção de sedimento em áreas comerciais é muito maior que em áreas residenciais, o qual pode estar associado à força das atividades econômicas presentes e, também, pelo gradiente de poluição atmosférica sobre estas áreas. As superfícies associadas ao tráfego veicular apresentam uma alta produção de sedimentos (normalmente definidas em toneladas de sedimentos por unidade de longitude), em função do número e das características do parque automotor; situação que se torna mais crítica na medida em que a cidade converte-se em pólo de desenvolvimento, como é o caso das áreas metropolitanas (SUTHERLAND, 2003).

Tabela 1 - Produção de sedimentos em áreas de bacias urbanas

\begin{tabular}{|c|c|c|}
\hline Uso do solo & Área de estudo & $\begin{array}{c}\text { Taxa de produção } \\
\text { sedimentos }\end{array}$ \\
\hline \multirow{3}{*}{ Urbanização } & Residencial baixa densidade & $50 \mathrm{Kg} \mathrm{Ha}^{-1} \mathrm{ano}^{-1}$ \\
\hline & $\begin{array}{c}\text { Residencial densidade } \\
\text { moderada }\end{array}$ & $322 \mathrm{Kg} \mathrm{Ha}^{-1} \mathrm{ano}^{-1}$ \\
\hline & $\begin{array}{c}\text { Residencial alta densidade } \\
\text { Comercial }\end{array}$ & $\begin{array}{l}350 \mathrm{Kg} \mathrm{Ha}^{-1} \text { ano }^{-1} \\
805 \mathrm{Kg} \mathrm{Ha}^{-1} \mathrm{ano}^{-1}\end{array}$ \\
\hline Construção & Erosão da superfície & $970 \mathrm{Kg} \mathrm{Ha}^{-1} \mathrm{ano}^{-1}$ \\
\hline Ruas e estradas & $\begin{array}{c}\text { Superfícies pavimentadas } \\
\text { Superfícies em terra } \\
\text { Ruas em florestas }\end{array}$ & $\begin{array}{l}502 \mathrm{Kg} \mathrm{Ha}^{-1} \mathrm{ano}^{-1} \\
3.4 \mathrm{t} \mathrm{Km}^{-1} \mathrm{ano}^{-1} \\
36 \mathrm{t} \mathrm{Km}^{-1} \mathrm{ano}^{-1}\end{array}$ \\
\hline
\end{tabular}

Adaptada de: Nelson e Booth (2002)

A maioria das pesquisas atuais sobre a distribuição de poluição difusa associada à dinâmica dos sedimentos urbanos está baseada no diagrama da figura anterior, no qual, em áreas urbanas, a principal fonte de poluentes é o sedimento depositado nas áreas impermeáveis e nos sistemas de drenagem, o qual consegue atingir mediante diversos mecanismos de transporte os corpos de água, os quais acabam sendo poluídos. É por isso, que o entendimento da contribuição de cada fonte de poluentes associados a sedimentos em áreas urbanas torna-se uma justificativa para avançar na formulação de instrumentos que permitam incorporar a área de qualidade de sedimentos à gestão dos recursos hídricos urbanos (NELSON e BOOTH, 2002; BANERJEE, 2003; CHARLESWORTH et al., 2003; AL-KHASHMAN, 2004; McALISTER et al., 2005; DUZGOREN-AYDIN et al., 2006; POLETO e MERTEN, 2007; JARTUN et al., 2008).

Pesquisas desenvolvidas por POLETO e MERTEN $(2007 ; 2008)$ em centros urbanos do sul do Brasil, com características socioeconômicas típicas das zonas periféricas brasileiras mostram que os sedimentos suspensos em corpos de água urbanos estão apresentando um enriquecimento antropogênico em uma proporção de dois ou mais vezes superior às concentrações de referência locais (background) de metais pesados como o zinco, chumbo e cromo, não evidenciando uma vocação industrial da área de estudo. Sugerindo a existência da influência dos sedimentos urbanos transportados pelo escoamento superficial durante eventos de chuva.

Existe uma forte tendência em avaliar amostras de sedimentos coletadas nas ruas, pelo fato de que esse local fornece: alta representatividade da atividade econômica predominante, facilidade no processo de amostragem e baixa probabilidade de alteração das propriedades química dos sedimentos por fatores ambientais. 


\section{TRANSPORTE DE SEDIMENTOS URBANOS}

O transporte de sedimentos em ambientes urbanos é complexo. Existe um pobre entendimento das rotas que as partículas de sedimentos utilizam desde sua fonte até corpos de água receptores, da taxa de transporte de sedimentos, da localização de sumidouros a curto e longo prazo e de como estas rotas impactam sobre o destino e a distribuição em longo prazo de poluentes no ambiente urbano (TAYLOR, 2007). O movimento dos sedimentos através do ambiente urbano pode ser representado mediante um diagrama de fluxos (Figura 3) conhecido como diagrama em cascata dos sedimentos urbanos (CHARLESWORTH e LEES, 1999).

Este diagrama reconhece a relação entre fontes, mecanismos de transporte e deposição de sedimentos. Taylor (2007) define este diagrama como um sistema altamente dinâmico. No topo do diagrama se considera que estão as fontes de sedimentos urbanos. A deposição de sedimentos sobre ruas é predominantemente de natureza transitória, com a remobilização tomando conta da parte baixa do diagrama. De forma global, o sedimento em ambientes urbanos é transportado pela ação da água, porém, existe uma redistribuição local sobre as superfícies impermeáveis devido à ação do vento (TAYLOR, 2007).

Segundo Deletic et al. (1997) o carregamento dos poluentes (associados às partículas de sedimentos) das superfícies é causado pela precipitação e pelo escoamento, etapas que ocorrem acima da superfície. Sequencialmente, as águas poluídas seguem para os sistemas de drenagem, onde os seguintes processos podem ocorrer: deposição dos sedimentos no leito da boca de lobo, suspensão dos sedimentos no fluido ou carreamento dos sedimentos dependendo da taxa de escoamento e do peso da partícula (DOTTO, 2006).

Além das superfícies impermeáveis, os locais de maior deposição de sedimentos em ambientes urbanos são as bocas coletoras (bocas de lobo) e interceptores de águas pluviais, rios, canais e lagos. Segundo Dotto (2006), o processo de acumulação de sedimentos em superfícies impermeáveis abarca toda uma variedade de processos típicos de tempo seco que ocorrem entre os eventos de precipitação, incluindo fenômenos como deposição, erosão devido à ação do vento, processo de limpeza das ruas, etc. Cada um destes processos induz a uma acumulação de sólidos na superfície e também de outros poluentes que são carreados durante os eventos de precipitação.

Os depósitos de sedimentos em rios, canais e lagos geralmente são removidos por engenharia aplicada (dragado). Estes sedimentos frequentemente atingem corpos de água, onde com o tempo o acumulo pode ocasionar impactos físicos e químicos sobre a qualidade da água e dos ecossistemas (TAYLOR, 2007).

Existe certa dificuldade na hora de interpretar os resultados obtidos no estudo da poluição difusa associada aos sedimentos urbanos quando se estudam os componentes de forma individual. A razão é, simplesmente, pelo fato de que alguns dos processos envolvidos na dinâmica não estão interligados entre si, o que não permite a visão holística da problemática (CHARLESWORTH e LEES, 1999).

Nesse sentido, a representação do movimento da partícula de sedimento em ambientes urbanos, definida na figura 3, é uma aproximação bastante útil no entendimento do fenômeno do transporte de poluentes adsorvidos nestes sedimentos, o qual permite obter mais informação de resultados como o citado por Butler e Clark (1995), quando menciona que partículas $<43 \mu \mathrm{m}$ coletadas em bocas coletoras são responsáveis da contribuição de $25 \%$ da demanda de oxigênio, $30 \%$ de nitrato e $50 \%$ de metais pesados em corpos da água de algumas cidades dos Estados Unidos. 


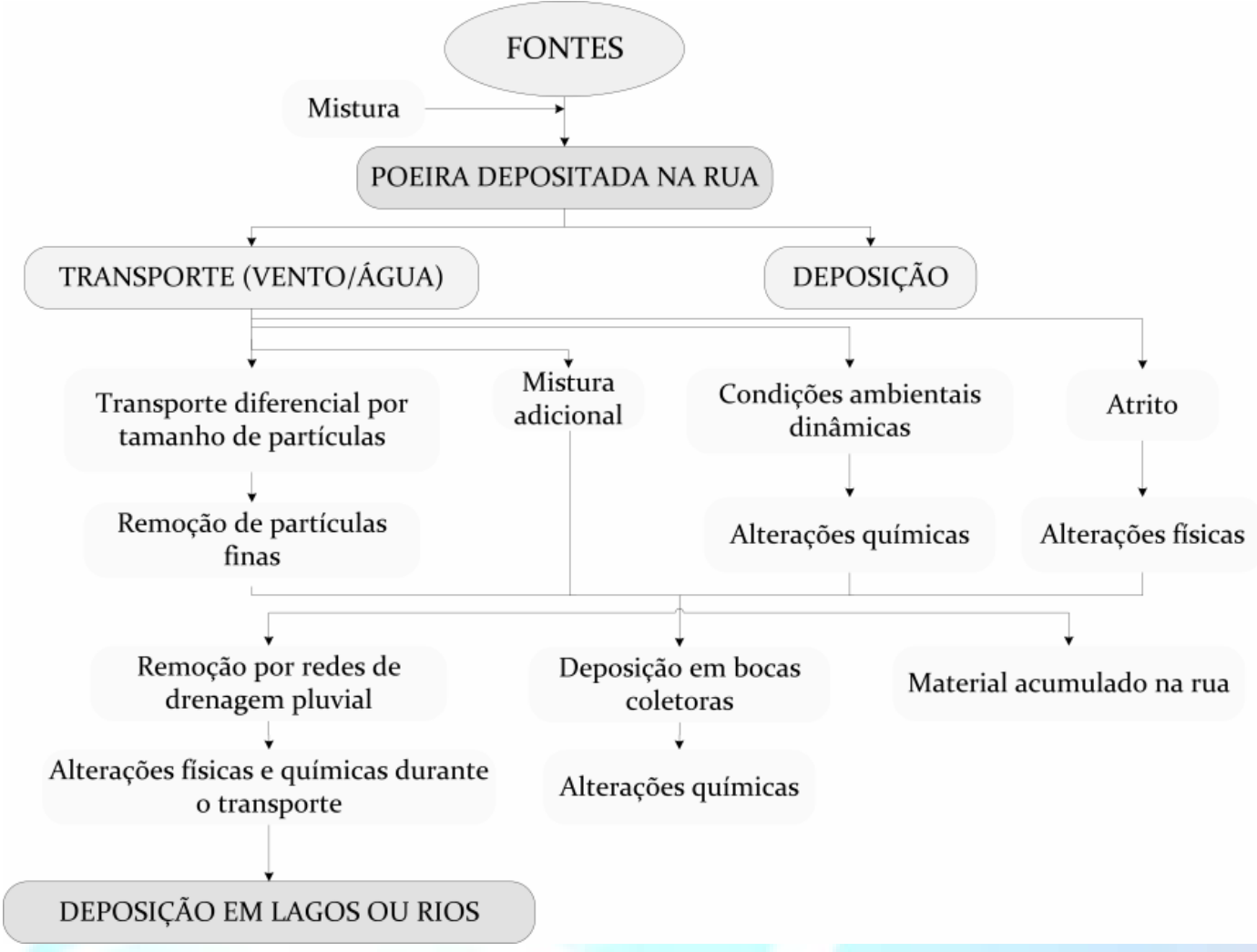

Figura 3. Movimento do sedimento nas bacias urbanas. Adaptado de: Charlesworth e Lees (1999)

Charlesworth e Lees (1999) em estudos realizados na cidade de Coventry (Reino Unido) encontraram que a tendência nesses locais é que os depósitos de sedimentos apresentem as concentrações mais elevadas de cádmio, cobre e chumbo, enquanto que as concentrações mais elevadas de zinco e de níquel estão nos sedimentos transportados. Nestes últimos, o comportamento pode estar associado com a solubilidade dos metais, facilitando seu transporte em solução e evitando que sejam alterados por processos inerentes ao tamanho da partícula como, por exemplo, limpeza da rua, atrito, ou transporte seletivo de um material mais fino durante eventos de precipitação. Pode igualmente estar associado com sua fonte, a qual pode ser menos suscetível de ser transportada em comparação com as partículas atmosféricas.

\section{METAIS PESADOS EM AMBIENTES URBANOS}

Uma das consequências do crescimento industrial desordenado é a grande liberação de íons e compostos metálicos no meio ambiente, causando danos à vida animal e vegetal. Desta forma, apesar dos bens minerais contribuírem para o desenvolvimento industrial, eles também se constituem como fontes poluidoras do ambiente, podendo provocar impacto ambiental, em alguns casos, bastante relevantes (OLIVEIRA, 2003). Esta apreciação também foi objeto de comentários por Callender e Rice (2000), Sorme e Lagerkvist (2002), Rule et al. (2006), Poleto e Castilho (2008) e Poggio et al. (2009).

Metais pesados são os poluentes do solo e do sedimento mais estudados por causa da sua toxicidade e persistência ainda que em baixas concentrações (POGGIO et al., 2009). Durante as três últimas décadas, têm sido executados extensos estudos em torno da presença de $\mathrm{Pb}, \mathrm{Cd}, \mathrm{Cu}$ e $\mathrm{Zn}$ e seus impactos no ambiente e à saúde humana (DUZGOREN-AYDIN et al., 2006). 
A importância ecológica dos metais pesados está relacionada ao seu potencial de acumulação e toxicidade, uma vez que tais elementos não são biodegradáveis e que a maioria podem ser tóxicos para animais e seres humanos, caso suas concentrações sejam suficientemente elevadas. Toxicidade é função de vários fatores, que incluem o tipo de organismo, biodisponibilidade de um metal ou elemento-traço no ambiente e seu potencial de bioacumulação na cadeia trófica (OLIVEIRA, 2003).

Em ambientes urbanos, os metais pesados são parte de nossas atividades cotidianas e muitos deles entram no ambiente urbano como subprodutos de atividades econômicas consideradas típicas em cidades em crescimento. Na Tabela 2 é apresentado o resumo de algumas das fontes antrópicas de metais pesados mais comuns em ambientes urbanos.

Tabela 2 - Principais fontes antrópicas de metais em ruas urbanas

\begin{tabular}{cl}
\hline Constituinte & \multicolumn{1}{c}{ Fonte } \\
\hline Chumbo & Escapamentos de veículos, pneus, óleo lubrificante, gordura, peças soldadas e tintas. \\
Zinco & Pneus, óleo de motor, gordura e freios. \\
Cobre & Galvanizado de metais, uso de pneus, peças de veículos, freios e inseticidas. \\
Cádmio & Pneus, escapamento de veículos e inseticidas. \\
Ferro & Corrosão de veículos, estruturas de aço e partes do motor. \\
Cromo & Galvanizado de metais, partes do motor e freios. \\
Níquel & Combustível diesel, óleo lubrificante, galvanizado de metais, freios e pavimento \\
& asfáltico. \\
Manganês & Pneus e freios \\
\hline
\end{tabular}

Adaptado de: Charlesworth e Lees (1999); Callender e Rice (2000); Sorme e Lagerkvist (2002); Rule et al. (2006); Poleto e Merten (2008); Poggio et al. (2009)

Para entender o comportamento das fontes de metais pesados associados a sedimentos em ambientes urbanos e predizer os caminhos e mobilidade dos poluentes e seus impactos, é preciso conhecer informações da sua geoquímica, a mineralogia (ROBERTSON et al., 2003; ADACHI e TAINOSHO, 2004) e a proporção do tamanho destas partículas no sedimento (CHARLESWORTH e LEES, 1999; NELSON e BOOTH, 2002; SUTHERLAND, 2003; BORTOLUZZI e POLETO, 2006). Entre os mecanismos mais comuns de associação de sedimentos - poluentes encontram-se: sorção de cátions metálicos, sorção de anions, complexação de metais na matéria orgânica e precipitação de íons inorgânicos.

\section{DISTRIBUIÇÃO DOS SEDIMENTOS E DOS METAIS EM ÁREAS IMPERMEAVEIS}

A distribuição do tamanho das partículas é um parâmetro fundamental para a caracterização de materiais heterogêneos. Técnicas granulométricas são empregadas para a caracterização de materiais com diversas origens, como industriais, fármacos, químicos, alimentares, como também em solos e sedimentos (BORTOLUZZI e POLETO, 2006). Em sedimentos, a análise do tamanho das partículas auxilia os estudos sobre agregação de partículas, transporte de sedimentos em rios, dissolução de partículas finas (STUMM e MORGAN, 1996) e análises de fontes de sedimentos urbanos (AL-RAJHI et al., 1995; GHANI et al., 2000; BANERJEE, 2003; CHARLESWORTH et 
al., 2003; SUTHERLAND, 2003; DUZGOREN-AYDIN et al., 2006; ZAFRA et al., 2007; JARTUN et al., 2008).

A separação de um material heterogêneo em quatro classes é a mais comum para fins de classificação da distribuição do tamanho de partículas. Para sedimentos, conforme Mudroch e Azcue (1995) dividem-se nas seguintes frações:

- $\quad$ Cascalho: $>2 \mathrm{~mm}$;

- $\quad$ Areia: $2 \mathrm{~mm}-63 \mu \mathrm{m}$;

- $\quad$ Silte: $63 \mu \mathrm{m}-2 \mu \mathrm{m}$; e,

- $\quad$ Argila: $<2 \mu \mathrm{m}$.

Podem-se ter subdivisões para uma maior precisão ou, conforme Bortoluzzi e Poleto (2006), estabelecer, inclusive, de forma arbitrária a classe de interesse, dependendo dos objetivos da pesquisa. Para sedimentos urbanos a distribuição de tamanhos de partículas é influenciada por muitos fatores, associados, basicamente, ao uso do solo.

Existe suficiente evidência que demonstra o fato de que sedimentos são enriquecidos com metais pesados, sobretudo, na fração fina das partículas. De forma similar aos sedimentos em outros ambientes, o aumento na carga de poluentes em partículas de tamanhos mais finos é geralmente associado ao incremento da área superficial nas partículas (ASE) de menor tamanho, fornecendo maior espaço para a adsorção de metais em argilominerais ou na matéria orgânica presente nas partículas de sedimento. $\mathrm{O}$ entendimento de que as cargas de metais pesados estão heterogeneamente distribuídas é importante na formulação de estratégias de gerenciamento e controle da poluição (TAYLOR, 2007).

Em termos de pesquisas associadas ao transporte de poluentes os resultados são diversos; Charlesworth e Lees (1999) relatam que é importante definir a distribuição de metais pesados em sedimentos coletados em ruas em função dos processos fonte-transporte-deposição através da análise granulométrica, normalmente considerando partículas $<2 \mathrm{~mm}$ como aquelas que são transportadas como cargas suspensas em típicos corpos de água urbanos e as partículas $<63 \mu \mathrm{m}$ representando os sedimentos que são acumulados em lagos e que estão associados com a adsorção de metais pesados (SUTHERLAND, 2003).

Charlesworth et al. (2003) conclui que, em superfícies urbanas de Coventry (Reino Unido), a fração dominante (maior transportadora de metais pesados) era a $<63 \mu \mathrm{m}$. Embora, a maior variabilidade (maior amplitude máximos e mínimos) em concentrações tenha sido encontrada na fração correspondente às partículas $<2 \mathrm{~mm}$. Sutherland (2003) também encontrou que a fração < $63 \mu \mathrm{m}$ foi predominante entre um grupo de frações $<1000 \mu \mathrm{m}$ avaliadas dentro de um grupo de amostras de sedimentos coletados em estradas, apresentando 38\% da massa total dos sedimentos armazenados nesse grupo. Nessa fração, encontrou-se que a concentração de chumbo (222 mg. $\left.\mathrm{kg}^{-1}\right)$, representava ao $51 \%$ da carga total de chumbo encontrada nos sedimentos coletados nas estradas monitoradas. ZAFRA et al. (2007) determinaram que a fração com maiores concentrações em todos os metais estudados foi a $<63 \mu \mathrm{m}$, exceto para o cobre, onde a máxima concentração foi obtida na fração compreendida entre $250-500 \mu \mathrm{m}$.

Poleto et al. (2009), em estudos que vem sendo realizados em 20 municípios do estado do Rio Grande do Sul - Sul do Brasil, sobre o monitoramento dos sedimentos urbanos coletados em ruas, avenidas e bueiros; mostra alguns resultados relacionados. A distribuição granulométrica encontrada nos sedimentos das ruas e avenidas (Figura 4a) apresenta um comportamento bimodal com um pico na fração menor a $100 \mu \mathrm{m}$ correspondente a areia muito fina segundo a classificação de Mudroch e Azcue (1995), podendo-se apreciar também a pouca variabilidade dos dados (baixos desvios padrões). Enquanto à distribuição granulométrica dos sedimentos em bueiros (Figura 4b) apresenta um comportamento unimodal, com a fração predominante sendo aquela entre 250 - 300 $\mu \mathrm{m}$ (areia mediana), confirmando alguns resultados obtidos por Butler e Clark (1995) em estudos 
realizados em bueiros. Destaca-se a alta variabilidade dos dados obtidos em contraste à distribuição granulométrica dos sedimentos coletados em ruas e avenidas.

Embora a distribuição de partículas seja importante na análise de resultados deste tipo de estudo, esta não se constitui em uma fonte única de explicação dos fenômenos de sorção de metais em sedimentos, basicamente pelo fato de que as partículas de sedimentos não estão isoladas do ambiente urbano, e são governadas por fatores físicos (topografia, direção do vento, escoamento superficial) e por fatores químicos $(\mathrm{pH}$, estrutura química da superfície impermeável, potencial redox).
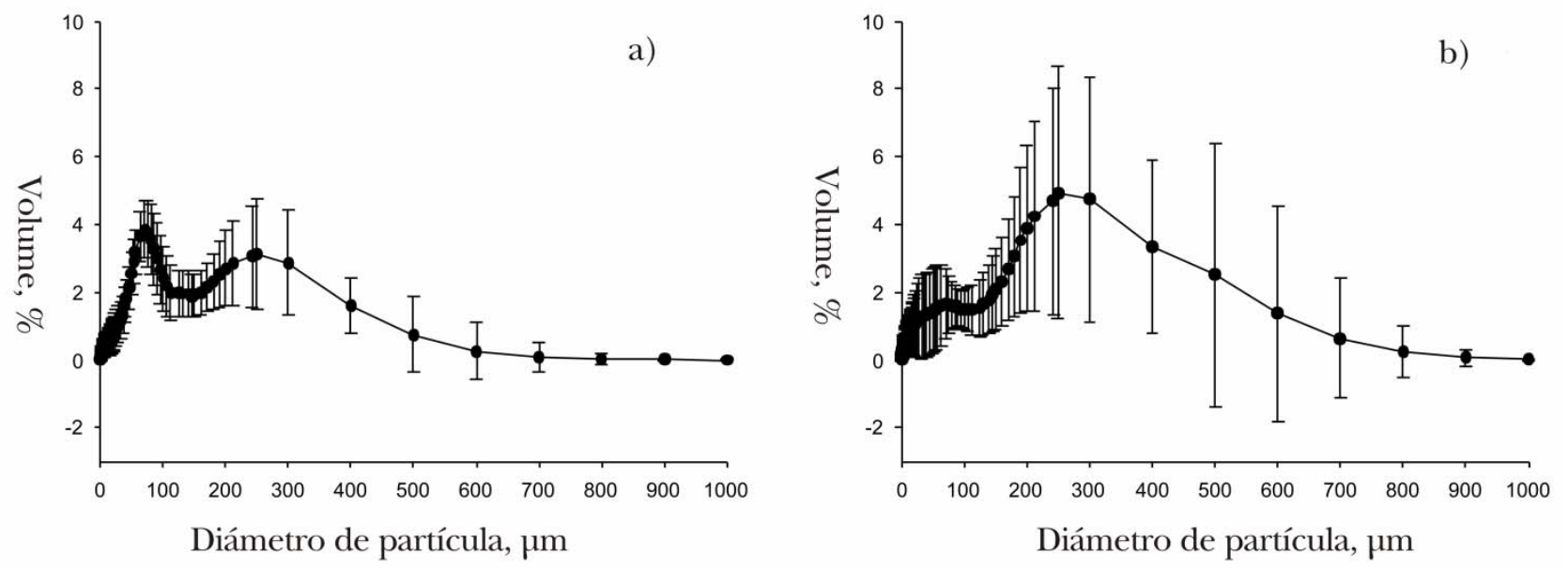

Figura 4. Tamanhos de partículas em centros urbanos de RS. a) Sedimentos de ruas; b) sedimentos de bueiros.

Adaptado de: Poleto et al. (2009)

Uma das razões pela qual às vezes é difícil de comparar e interpretar distribuições de metais pesados em uma área urbana está relacionada com o tempo de permanência do sedimento em um mesmo local.

Duzgoren-Aydin et al. (2006), fazem referência a este fator no seu estudo, onde obtiveram concentrações diferentes de metais em amostras coletadas em ruas de uma mesma área. As explicações dos resultados apontam à influência da frequência de limpeza das superfícies, quer dizer, o sedimento ficava muito mais tempo acumulado, circunstância que facilitava a interação entre os metais presentes nos sedimentos e as condições ambientais do meio onde se encontravam.

Charlesworth et al. (2003) concluíram que os resultados obtidos na comparação de distribuição de metais pesados entre as cidades de Birmigham e Coventry, Inglaterra, apresentam uma margem de incerteza devido ao desconhecimento do histórico do tempo de residência dos sedimentos nos locais amostrados.

\section{CONSIDERAÇÕES FINAIS E DESAFIOS FUTUROS}

É evidente que existe certa preocupação em torno do aumento das cargas de poluentes desde os centros urbanos até os corpos de água receptores e que ainda existem muitas incertezas no estudo dos fatores relacionados com a interação entre as partículas de sedimentos, os poluentes e o ambiente. Duzgoren-Aydin et al. (2006), reconhece que a maioria dos estudos associados com a qualidade das águas em sistemas de drenagem urbana estão localizados em países do primeiro 
mundo e que a pesquisa ainda encontra-se muito limitada em países em desenvolvimento, onde basicamente ainda trabalha-se na quantificação e previsão de vazões.

Atualmente se considera o sedimento como um recurso de interesse econômico, social e ecológico que precisa de um gerenciamento sustentável, onde se articulem o entendimento das propriedades físicas e químicas dos sedimentos em ambientes urbanos com informações ecológicas e hidrológicas dos corpos de água receptores em uma escala regional e, assim, avaliar os possíveis cenários de poluição dos recursos hídricos. Essas informações servirão de suporte em processos de tomada de decisões para o controle da poluição (TAYLOR, 2007).

Já em matéria de manejo e remediação de sedimentos, uns dos desafios mais importantes neste momento é a implementação de sistemas sustentáveis de drenagem urbana que permitam resolver os problemas mais críticos relacionados com o manejo dos sedimentos em centros urbanos. Os quais devem responder as seguintes interrogações:

- Como realizar a retenção e a retirada adequada desses sedimentos de modo que estes não aportem nos corpos de água?

- Como controlar a liberação dos poluentes sorvidos nos sedimentos após a sua retenção no sistema?

- Como transportar e onde dispor esse material contaminado?

Da mesma forma, torna-se necessária a implementação de programas de pesquisa contínuos sobre a dinâmica dos poluentes associados aos sedimentos, procurando aumentar o conhecimento de algumas áreas, como, por exemplo:

- Avaliação do impacto dos sedimentos urbanos;

- Definição de padrões de qualidade e possíveis tratamentos de sedimentos dragados;

- Avaliação de riscos associados à poluição por sedimentos utilizando bioensaios;

- Exploração de ferramentas SIG, para gerar informações cartográficas temáticas que possam ser utilizadas na interpretação da distribuição espacial dos poluentes.

\section{CONCLUSÕES}

De uma forma geral, apesar dos sedimentos urbanos serem encontrados em diferentes contextos na literatura científica e, no passado, ter sido feita referência especialmente aos sedimentos acumulados nas superfícies impermeáveis das ruas pavimentadas, pela conceituação moderna, estes representam qualquer sedimento encontrado dentro de um ambiente urbano (POLETO, 2007). Portanto, esses sedimentos compreendem várias fontes potenciais, tais como ruas, sistemas de drenagem e os próprios corpos de água.

As partículas de sedimentos urbanos geram impactos negativos ao ambiente, entre os quais, encontram-se: impactos na qualidade dos recursos hídricos, redução da capacidade de transporte em sistemas de drenagem, incremento na concentração de partículas atmosféricas e redução na profundidade de cursos de água navegáveis. Portanto, o gerenciamento dos sedimentos é um elemento chave na concepção de ambientes urbanos sustentáveis.

A natureza recente das informações relacionadas com o transporte de poluentes adsorvidos em sedimentos urbanos é um fator limitante na identificação de mudanças temporais em resposta a fatores internos ou externos. Portanto, é preciso gerar uma linha base de informações que permitam predizer mudanças futuras dentro do ambiente urbano. Razão que justifica continuar com as pesquisas nesta área nos centros urbanos brasileiros. 


\section{AGRADECIMENTOS}

Os autores agradecem ao CNPq e a Capes pelo apoio.

\section{REFERÊNCIAS}

ADACHI, K.; TAINOSHO, Y. Characterization of heavy metal particles embedded in tire dust. Environment International, n. 30, 2004. p. 1009-1017.

AL-KHASHMAN, O. Heavy metal distribution in dust, street dust and soils from the work place in Karak Industrial Estate, Jordan. Atmospheric Environment, n. 38, p. 6803 - 6812, 2004.

AL-RAJHI, M. A.; AL - SHAYEB, S. M.; SEAWARD, M. R.; EDWARDS, H. G. Particle size effect for metal pollution analysis of atmospherically deposited dust. Atmospheric Environment, v. 30, n. 1, p. $145-163,1995$.

BANERJEE, A. D. K. Heavy metal levels and solid phase speciation in street dusts of Delhi, India. Environmental Pollution, n. 23, p. 95-105, 2003.

BORTOLUZZI, E. C.; POLETO, C. Metodologias para estudos de sedimentos: ênfase na proporção e a natureza mineralógica das partículas. In: POLETO, C; MERTEN, G. (Org) Qualidade dos Sedimentos. Porto Alegre: ed. ABRH, p. 83-140, 2006.

BUTLER, D.; CLARK, P. Sediment management in urban drainage catchments. Construction Research and Information Association, CIRIA Report 134. London, 1995.

CALLENDER, E.; RICE, K. Rice. The Urban Environmental Gradient: Anthropogenic Influences on the Spatial and Temporal Distributions of Lead and Zinc in Sediments. Environmental Science \& Technology, v. 34 n .2, p. $232-238,2000$.

CHARLESWORTH, S. M.; EVERETT, M.; McARTHY, R.; ORDOÑEZ, A.; MIGUEL, E. A comparative study of heavy metal concentration and distribution in deposited street dusts in a large and a small urban area: Birmingham and Coventry, West Midlands, UK. Environment International, n. 29, p. $563-573,2003$.

CHARLESWORTH, S. M.; LEES, J. A. Particulate-associated heavy metals in the urban environment: their transport from the source to deposit, Coventry, UK. Chemosphere, v. 39, n.5, p. 833-848, 1999.

DELETIC, A.; MAKSIMOVIC, C.; IVETIC, M. Modelling of storm wash-off of suspended solids from impervious surfaces. Journal of Hydraulic Research, Vancouver, v. 35, n. 1, p. 99-118, 1997.

DOTTO, C. B. Acumulação e balanço de sedimentos em superfícies asfálticas em área urbana de Santa Maria - RS. Santa Maria. 2006. 126 f. Dissertação (Mestrado em Engenharia Civil) Universidade Federal de Santa Maria, Santa Maria. RS. 2006. 
DUZGOREN-AYDIN, N. S.; WONG, C. S.; AYDIN, A.; SONG, Z.; YOU, M.; LI, X. D. Heavy metal contamination and distribution in the urban environment of Guangzhou, SE China. Environmental Geochemistry and Health, n. 38, p. 375 - 391, 2006.

FEDERAL INTERAGENCY STREAM RESTORATION WORKING GROUP. Federal Interagency Stream Restoration Working Group, Stream Corridor Restoration: Principles, Processes, and Practices, NTIS, Department of Commerce, Springfield, VA. 1998.

GELLIS, A.; SMITH, S.; STEWART, S. Watershed sediment sources. In: LANGLAND, M.; CRONIN, T. (Org.) A Summary Report of Sediment Processes in Chesapeake Bay and Watershed: Water-Resources Investigations Report 03-4123. New Cumberland - Pennsylvania: US Geological Survey Publications, p. 29 - 33, 2003. Disponível em http://www.mgs.md.gov/coastal/pub/wrir034123.pdf. Acesso em: 28 mar. 2009.

GHANI, A.; ZAKARIA, N.; KASSIM, M.; NASIR, B. A. Sediment size characteristics of urban drains in Malaysian cities. Urban Water, n. 2, p. 335 - 341, 2000.

JARTUN M.; OTTESENA, R.; STEINNESB, E.; VOLDENA, T. Runoff of particle bound pollutants from urban impervious surfaces studied by analysis of sediments from stormwater traps. Science of the Total Environment, n. 396, p. 147 - 163, 2008.

\section{MARTINEZ, L. L. G. Distribuição de Poluição Difusa por Sedimentos Urbanos em Áreas} Impermeáveis em Porto Alegre. 2010. 126 f. Dissertação (Mestrado em Recursos Hídricos e Saneamento Ambiental) - Instituto de Pesquisas Hidráulicas - Universidade Federal do Rio Grande do Sul, Porto Alegre, RS. 2010.

McALISTER, J.; SMITH, B.; BAPTISTA, J.; SIMPSON, J. Geochemical distribution and bioavailability of heavy metals and oxalate in street sediments from Rio de Janeiro, Brazil: a preliminary investigation. Environmental Geochemistry and Health, n. 27, p. 427 - 441, 2005.

MUDROCH, A.; AZCUE, J. M. Manual of aquatic sediment sampling. Florida, EUA: CRC press, 1995. $219 \mathrm{p}$.

NELSON, E.; BOOTH, D. Sediment sources in an urbanizing, mixed land-use watershed. Journal of Hydrology, n. 264, p. $51-68,2002$.

OLIVEIRA, J. A. Diagnóstico ambiental do solo e sedimento do Parque Estadual Turístico do Alto Ribeira (PETAR). 2003.116 f. Dissertação (Mestrado em Ciências - Química Analítica) Universidade Federal de São Paulo, São Carlos. SP. 2003.

PEREIRA, J. A.; BAPTISTA-NETO, J.; SMITH, B. J.; McALLISTER, J. J. The contribution of heavy metal pollution derived from highway runoff to Guanabara Bay sediments - Rio de Janeiro/ Brazil. Anais ... Rio de Janeiro: Academia Brasileira das Ciências, v. 79, n. 4, , 2007, p. 739 - 750.

POGGIO, L.; VRSCAJ, B.; SHULIN, R.; HEPPERLE, E.; AJMONE, F. Metals pollution and human bioaccessibility of topsoils in Grugliasco (Italy). Environmental Pollution, v.157, p. 680 689, 2009. 
POLETO, C. Fontes potenciais e qualidade dos sedimentos fluviais em suspensão em ambiente urbano. 137 f. 2007.Tese (Doutorado em Recursos Hídricos e Saneamento Ambiental) - Instituto de Pesquisas Hidráulicas,Universidade Federal do Rio Grande do Sul, Porto Alegre. RS. 2007.

POLETO, C.; BORTOLUZZI, E.; CHARLESWORTH, S.; MERTEN, G. Urban sediment particle size and pollutants in Southern Brazil. Journal of Soils and Sediments, v. 9, p. 317 - 327, 2009.

POLETO, C.; CASTILHO, Z. C. Impacto por poluição difusa de sedimentos em bacias urbanas. In: POLETO, C. (Org.) Ambiente e Sedimentos. Porto Alegre: Ed. ABRH, p. 193 - 227, 2008.

POLETO, C.; CHARLESWORTH, S. Sedimentology of Aqueous Systems. Londres: WileyBlackwell, 2010. 216p.

POLETO, C.; MERTEN, G. H. Estudos de Zn e Ni em sedimentos fluviais em suspensão e o risco potencial aos recursos hídricos. Revista Brasileira de Recursos Hídricos, v. 13, p. 147-154, 2008.

POLETO, C.; MERTEN, G. H. Urban watershed studies in southern Brazil. Journal of Urban and Environmental Engineering, v.1, n.2, p. 70-78, 2007.

PORTO, R.; ZAHED, K.; TUCCI, C.; BIDONE, F. Drenagem Urbana. In: TUCCI, C.E.M. (Org.) Hidrologia: Ciência e Aplicação. Porto Alegre: Ed. Universidade Federal do Rio Grande do Sul, p.805-847, 2004.

ROBERTSON, D. J.; TAYLOR, K. G.; HOON, S. R. Geochemical and mineral magnetic characterization of urban sediment particulates, Manchester, UK. Applied Geochemistry, n. 18, p. $269-282,2003$.

RULE, K. L.; COMBER, S. D. W.; ROSS, D.; THORTON, A.; MAKROPOULOS, C. K.; RAUTIU, R. Diffuse sources of heavy metals entering an urban wastewater catchment. Chemosphere, n. 63, p. $64-72,2006$.

SORME, L.; LAGERKVIST, R. Sources of heavy metals in urban wastewater in Stockholm. The Science of the Total Environment, n. 298, p. 131-145, 2002.

STUMM, W.; MORGAN, J. J. Aquatic Chemistry: Chemical equilibria and rates in natural waters. 3 ed. New York: Jhon Wiley \& Son, 1996. 1022 p.

SUTHERLAND, R. Lead in grain size fractions of road-deposited sediment. Environmental Pollution, n. 121, p. $229-237,2003$.

TAYLOR, K. Urban Environments. In: TAYLOR, K.; PERRY, C. Environmental Sedimentology. Manchester: Blackwell, p. 191 - 222. 2007.

ZAFRA, C.; TEMPRANO, J.; TEJERO, J. I. Contaminación por escorrentía superficial urbana: metales pesados acumulados sobre la superficie de una via.Ingeniería e Investigación, v. 27, n. 1, p. $4-10,2007$. 